\title{
Effects of semi-rigid ankle orthoses on tasks related to athletic performance following a bout of fatiguing exercise
}

\author{
James A. Yaggie ${ }^{1}$, W. Jeffrey Armstrong ${ }^{2 *}$, Christina Smith ${ }^{3}$, Andrew Miller ${ }^{3}$, \\ Rebekah Trimbach ${ }^{3}$ \\ ${ }^{1}$ Department of Health and Human Performance, The University of Findlay, Findlay, USA \\ ${ }^{2}$ Department of Health \& Physical Education, Western Oregon University, Monmouth, USA; \\ *Corresponding Author: armstrong.wou@gmail.com \\ ${ }^{3}$ Department of Physical Therapy, The University of Findlay, Findlay, USA
}

Received 1 October 2013; revised 1 November 2013; accepted 12 November 2013

Copyright (C) 2013 James A. Yaggie et al. This is an open access article distributed under the Creative Commons Attribution License, which permits unrestricted use, distribution, and reproduction in any medium, provided the original work is properly cited.

\section{ABSTRACT}

Despite the perceived protection of semi-rigid ankle orthoses (SRAO), coaches and clinicians are often concerned with the restriction that the SRAO may pose on functional performance. The purpose of this study was to determine the effect of SRAO on performance-selected tasks, following fatiguing activity. Twenty active, college-age subjects $(22.7 \pm 1.2$ years; weight $71.7 \pm$ $13.0 \mathrm{~kg}$; height $171.7 \pm 9.4 \mathrm{~cm}$ ), without lower extremity trauma within 2 years, consented participation. Randomized trials for balance (star excursion balance test (SEBT)), agility (shuttle run (SR)), and power (vertical jump (VJ)) were compared between 4 conditions [fatigued, braced (FB); fatigued, un-braced (FUB); nonfatigued, braced (NFB); non-fatigued, un-braced (NFUB)]. Participants were bilaterally fitted with a well-known, commercially available SRAO for all braced trials. Two Wingate supramaximal exercise bouts were used as the fatiguing protocol. RMANOVA was performed for each functional test to determine main effects (SPSS, IL; $\alpha=0.05$ for all tests). No main effects for VJ \& SR were observed under any of the bracing conditions. Main effect for bracing was observed for the SEBT. Tukey pairwise comparisons revealed that there were significant increases in distance reached for the posterior-medial direction (NFB $=0.91 \mathrm{~cm} \pm 0.2 ; \mathrm{FB}=0.95 \pm 0.2 ; p=0.046)$ and medial direction (NFB $=0.74 \mathrm{~cm} \pm 0.1 ; \mathrm{FB}=0.79$ $\pm 0.1 ; p=0.039$ ) during the braced conditions. Although subjects reported that the SRAO re- stricted ROM during most SEBT directions involving inversion and eversion, overall performance was not hindered by the application of the brace. Further, the only main effects observed with functional performance involved increases in SEBT that could likely be attributed to comfort and confidence of motion while wearing an ankle device. It was concluded that articulated SRAO effectively limits ankle mobility, but does not appear to hinder athletic performance following a fatiguing bout.

Keywords: Agility; Ankle Bracing; Balance; Fatigue; Stability; Vertical Jump

\section{INTRODUCTION}

Athletes rely heavily on stability and balance for athletic performance. Unfortunately, the movements required in some sports leave athletes at risk for injury. A review conducted by Fong et al. [1] examined the distribution of injuries in athletics via meta-analysis and concluded that the ankle was the commonest body segment injured in sports [1]. Further, lateral ankle sprains can account for $15 \%$ to $30 \%$ of all injuries in sports [2]. Risk factors for such injuries include participation in sports that require jumping, landing, and cutting maneuvers, and the risk for injury is increased more with a history of previous ankle sprain(s) [3]. Moreover, the risk for injury may increase late in activity when the athlete becomes physically fatigued [4]. This is likely due to instability at the ankle and more proximal joints as the result of decreased proprioception [5,6].

Consequently, ankle bracing has become increasingly 
prevalent in sports [7-9] and has lead clinicians, coaches and athletes to utilize ankle strapping as a preventative measure. The objective of ankle bracing is to provide mechanical support at the joint by limiting inversion and eversion but not restrict dorsiflexion and plantarflexion, thus allowing the athlete to maintain function for performance [2]. Clinical methods to stabilize the ankle include the use of tape, ACE bandages, soft braces, semi-rigid braces, and rigid braces are available depending on the types of movement needed at the ankle for competition $[2,10]$. However, as the amount of stability increases at the joint, the mobility at the joint decreases, and most athletes have personal preferences for brace use and type. According to Rosenbaum [2], semi-rigid braces provide the most mechanical support at the ankle secondary to rigid braces but still retain functional medial to lateral mobility.

Among the types and styles of commercially available ankle orthoses, the Active Ankle ${ }^{\circledR}$ (Active Ankle Systems, Louisville, KY) is a lightweight, comfortable, durable brace, and has a quick-fit, single strap system that adjusts to both high and low top shoes. The brace offers flexibility, security, and is said to provide more support than the leading air-stirrup brace, the leading lace-up brace, and adhesive tape [11]. Although, Shaw et al. [12] found the lace-up brace performed better than the Active Ankle ${ }^{\circledR}$ brace when testing for dynamic stability using “Time to Stabilization”, others have found that the Active Ankle ${ }^{\circledR}$ provides a great deal of mechanical support.

Lindey et al. [13] examined functional range of motion of the ankle in semi-rigid braces and adhesive tapes. The three semi-rigid braces tested were the Active Ankle $^{\circledR}$, Airstirrup Ankle ${ }^{\circledR}$ (Aircast Inc., Summit, NJ) and Ankle Ligament Protector ${ }^{\circledR}$ (Donjoy Orthopedics, Carlsbad, CA). Out of the braces and adhesive tape, the Active Ankle ${ }^{\circledR}$ brace had the best maximum dorsiflexion, plantar flexion and functional range of motion [13].

Bot and van Mechelen [14] studied the impact ankle bracing on performance of vertical jump, running speed, agility, and broad jump. The authors concluded that ankle bracing has no effect on performance of those four athletic tasks [14]. However, the researchers did suggest that if the ankle joint range of motion (ROM) is restricted by the brace, athletic performance will diminish [14]. Similarly, Cordova et al. [7] conducted a metaanalysis assessing the effects of ankle braces on functional performance, as well as, the kinetics and kinematics of the joint. These results suggested that ankle bracing produces minimal to small decrements on lower extremity functional performance [7]. The authors did note negative effects on examining vertical jump performance when ankle plantar-flexion was restricted through a brace.

The impact of ankle instability becomes particularly noticeable when the muscles surrounding the joint be- come fatigued. Gribble et al. [6] noted that fatigue may impair proprioceptive and kinesthetic properties of the joints because fatigue increases the threshold of the muscle spindle discharge which disturbs afferent feedback thereby altering joint awareness. Without adequate muscle spindle reaction, the joint and associated structures are subject to excessive stretch and injury.

It is been hypothesized that ankle braces may improve dynamic stability with the onset of fatigue [12]. Shaw et al. [12] used force plates to determine if braces decrease time to stabilization in fatigued subjects. The results showed that both the lace-up ankle brace (Sweede- ${ }^{\circledR}$, Sweede-O, Inc., North Branch, MN) and the semi-rigid brace (Active Ankle ${ }^{\circledR}$ ) had a decrease in time to stabilization [12].

Statement of the Problem. Bracing may offer support to the ankle joint at a time when muscular support in impeded during the fatigued state. Further, the qualities and properties of ankle strapping may lead to a restriction of the joint with little impact on athletic performance. However, there is limited research investigating the functionality of a brace following exhausting bouts of lower extremity activity. The present study evaluated the effects on various athletic demands before and after fatigue, as well as in braced and unbraced conditions.

\section{METHODS}

Subjects. Twenty healthy, college age subjects (11 females and 9 males; mean age $=23 \pm 1$ years; mean weight $=71.7 \pm 13 \mathrm{~kg}$; mean height $=171.7 \pm 9.4 \mathrm{~cm}$ ) were recruited through convenience sampling and consented to participation through completion IRB approved informed consent documentation in accordance with the Declaration of Helsinki. All participants were recreationally active, participating in a minimum of 20 - 30 minutes of activity at least three times a week.

Exclusion criteria included acute lower extremity injuries, severe ankle injuries within the past two years or any lower extremity surgeries that would inhibit the current research study. Additionally, participants were excluded if they have any medical conditions, i.e., cardiovascular or vestibular, that could be exacerbated by the fatiguing protocol in this study.

Bracing. The Active Ankle ${ }^{\circledR} \mathrm{T}-2$ ankle orthosis (Cramer Products, Inc., KS, Figure 1) was applied bilaterally following the manufacturer's guidelines. Range of motion (ROM) measurements were taken at the beginning of each testing session and documented to note the restrictions created by the brace. On the days where the brace was worn, measurements were taken before and after brace application. Measurements were also taken at the end of each testing session in order to account for loosening of the brace. The motions measured included ankle dorsiflexion, plantarflexion, inversion, and ever- 


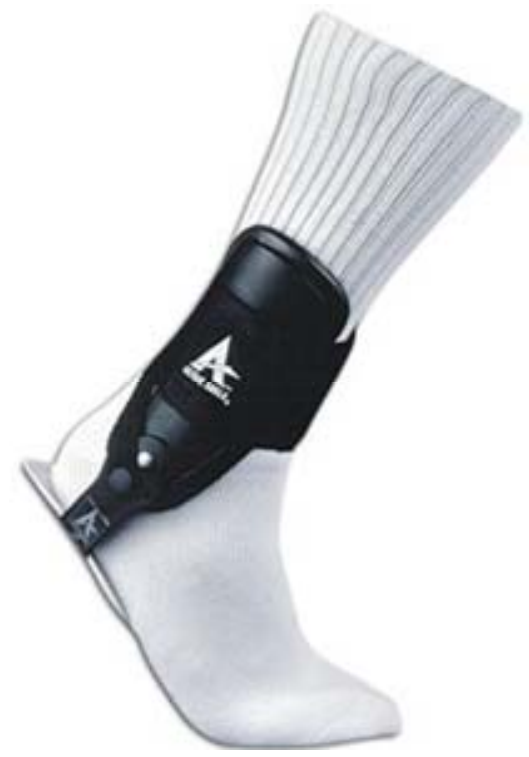

Figure 1. Active Ankle ${ }^{\circledR} \mathrm{T}-2$ brace.

sion. Measurement protocol followed the guidelines from Norkin and White [15]. The same researcher applied the brace and assessed ROM with the same 6-inch goniometer for each subject. The brace was reapplied if it did not produce appropriate restrictions. Once the brace was applied correctly, it was not adjusted during testing.

Fatigue Protocol. Subjects performed two Wingate (WAnT) supramaximal exercise bouts separated by a 2minute rest as the fatigue protocol. This was performed on a modified Monark cycle ergometer (Model \#858E, Stockholm, Sweden) at a resistance of 0.075 kilopounds per kilogram of body weight in order to standardize the fatigue method. Each WAnT was 30 seconds in length and was performed at maximal effort. For the purposes of this study the investigators chose to fatigue the entire lower extremity following WAnT protocol used by Yaggie and Armstrong [5]. Other studies have used localized fatigue protocols, however strictly fatiguing the ankle joint does not account for any compensation by more proximal joints and musculature [5]. Also, fatiguing the entire lower extremity more closely mimics the conditions that may occur within athletic competition. Using supramaximal WAnT exercise bouts Yaggie and Armstrong [5] found significant postural sway and instability post-fatigue. Furthermore, the WAnT uses an ergometer that requires concentric patterns of muscle activity to fatigue the muscle rather than eccentric muscle activity that has been shown to produce damage that further reduces forceful contractions and delays recovery [5].

Performance Tests. The subjects participated in tests of athletic performance on two non-consecutive days while wearing an ankle brace during fatigued and nonfatigued conditions on one day and the same tests were performed without the brace in fatigued and non-fatigued states on the other day. Half of the subjects wore the brace on the first day, while the other half of the subjects performed the tests without the brace. The order of performance testing [Countermovement Vertical Jump (CMJ), Shuttle Run (SR), and Star Balance Excursion Test (SEBT)] was randomized through a Balance Latin Square design.

The CMJ was performed following the guidelines set up by Brown and Ferrigno [16]. This tested the effect of ankle bracing and fatigue on the individual's power performance. A countermovement jump was performed with the subject standing with feet shoulder-width apart. The subject was instructed to quickly squat down, flexing hips and knees to $90^{\circ}$, to apply a pre-stretch to the muscles. The subject then forcefully jumped up, reaching up with the dominant hand with the aim of moving as many levers as possible. Subjects performed 3 jumps with oneminute rest time between trials, and the best attempt was recorded.

The 20-yard shuttle was also performed following the guidelines set up by Brown and Ferrigno [16]. This evaluated the effects of ankle bracing and fatigue on the individual's speed and agility performance. Subjects began with feet hip-width apart, straddling a starting line. Five-yards to the right and 5-yards to the left tape markers were in place for the individuals to touch. Subjects turned to the right, ran 5-yards and touched the line with his/her right hand. He/she then turn back to the left, ran 10 yards to the line passing the starting point, and touched the line with his/her left hand. Lastly, the subject turned back to the right and ran 5-yards through the start/finish line (Figure 2). The recorder started the stopwatch upon verbalizing "Go" and stopped it when the subject crossed the start/finish line at the end. To prevent inter-rater differences, the same researcher described and recorded results for each subject. The 3 trials were performed with a rest time of one minute between trials. Times were recorded and averaged together in an attempt to eliminate human error as there was risk for a time delay in the start and stop of a hand-held stopwatch.

The SEBT was incorporated to assess the subject's balance. The SEBT was set up with a line drawn in eight directions, with a 45-degree angle to the next direction

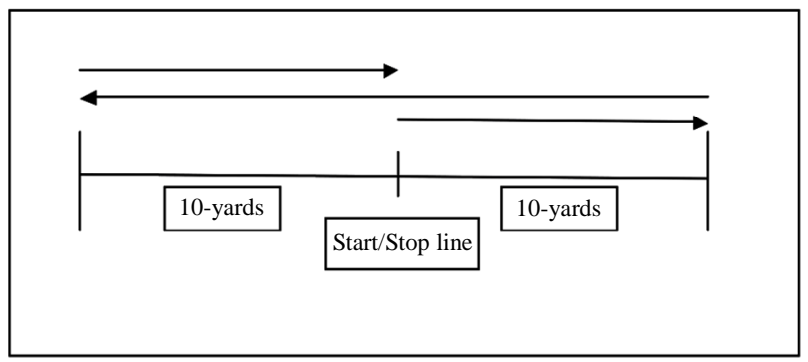

Figure 2. 20-yard shuttle run. 
(Figure 3). The subjects stood on their dominant leg at the intersection of the eight lines, while extending the opposite leg as far as possible in each of the eight directions: anterior, anterior-medial, medial, posterior-medial, posterior, posterior-lateral, lateral, and anterior-lateral directions [17]. Each subject completed the SEBT three times, performing a reach in all directions per trial. To prevent inter-rater differences, the same researcher marked and recorded the subjects' distance. The average distance in each direction was calculated. If the subject's foot touched the ground, a loss of balance was noted. A rest break of one minute between each set was allotted.

Following data collection, a RM-ANOVA was performed for each functional test to determine main effects of bracing and fatigue. If main effects were noted, pairwise comparisons were run to determine the specific differences in each functional performance. All tests were statistically scrutinized $(\alpha=0.05)$ using SPSS for Windows (V. 17, Chicago, IL).

The various testing conditions are abbreviated as follows:

non-fatigued braced $=$ NFB

non-fatigue unbraced $=$ NFUB

fatigue braced $=$ FB

fatigued unbraced $=$ FUB

\section{RESULTS}

Countermovement Jump Height (CMJH). Mean CMJH ( \pm S.D.) are reported in Table 1. On average, the height of the jump trials after fatigue were less than that of the non-fatigued trials, yet the values were not significantly different $(p=0.269$; power $=0.91)$. Bracing the ankle did not have a significant effect in either condition ( $p=0.329$ and $p=0.127$ for FB and FUB, respectively). Although, the mean jump height (47.59 \pm 15.6 $\mathrm{cm}$ ) for FB was higher than that of the FUB trials (45.61 $\pm 14.2 \mathrm{~cm}$ ) these numbers were not statistically signifi-

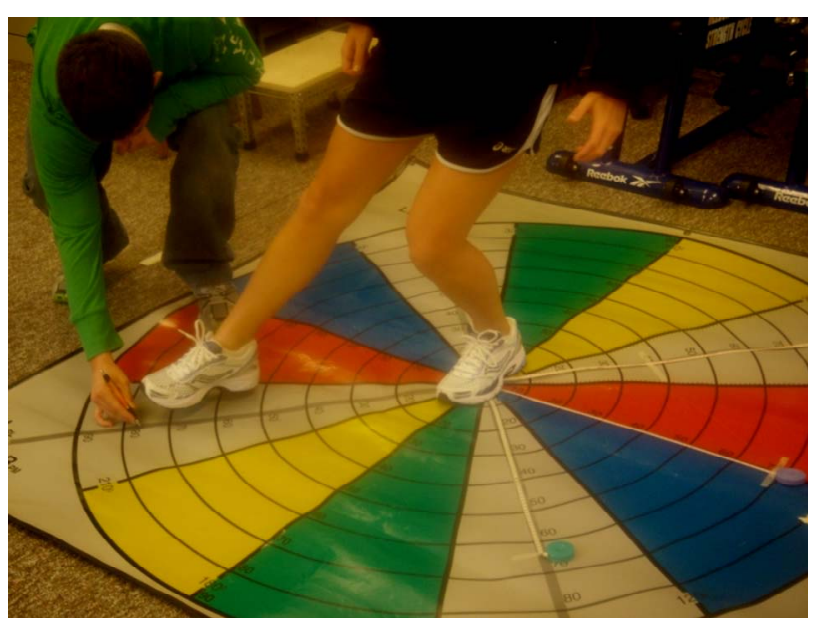

Figure 3. Performance of the SEBT. cant $(p=0.093)$. However, this may indicate that the bracing may attenuate the effects of fatigue. The NFUB jumps averaged $49.42 \pm 15.3 \mathrm{~cm}$ and NFB jumps averaged $48.17 \pm 16.4 \mathrm{~cm}$ with no significant difference to note.

Shuttle Run (SR). Mean SR scores ( \pm S.D.) are reported in Table 1. No significant difference existed between the two non-fatigued conditions in the amount of time it took to complete the 20-yard shuttle run ( $p=$ 0.632). The mean time for the subjects to complete the shuttle test during NFB conditions was $5.57 \pm 0.8 \mathrm{sec}-$ onds and the mean for NFUB conditions was $5.62 \pm 0.9$ seconds. There also lacked a significant difference between the fatigued conditions ( $p=0.377)$. The average time for FB trails was $6.02 \pm 0.9$ seconds while the mean for FUB trials was $5.89 \pm 0.8$ seconds. According to these data, it did not appear that wearing an ankle brace aided nor hindered performance under fatigued or rested conditions. However, the fatigued trials, as predicted, were consistently slower than all non-fatigued trials (NFUB v. FUB ( $p=0.038)$; NFB v. FB $(p=0.0010)$ ).

Star Balance Excursion Test (SEBT). Results of the SEBT are displayed above in Table 2. Fatigue trials differed from the non-fatigued trials (braced vs. unbraced conditions). There was no main effect in the following directions; Anterior, Anterior Lateral, Lateral, Posterior Lateral, Posterior, and Anterior Medial $(p \geq 0.05)$. There was a significant increase in Posterior Medial $(p=0.046)$ following the fatiguing bout. Independent $t$-tests were run on the data to assess these potential differences independent of the more homogenous conditions. The re-

Table 1. Countermovement Jump Height (CMJH) and Shuttle Run $(S R) \pm$ S.D.

\begin{tabular}{ccccc}
\hline & NFUB & FUB & NFB & FB \\
\hline CMJH (cm) & $49.42 \pm 15.3$ & $45.61 \pm 14.2$ & $48.17 \pm 16.4$ & $47.59 \pm 15.6$ \\
SR (s) & $5.62 \pm 0.9$ & $5.89 \pm 0.8$ & $5.57 \pm 0.8$ & $6.02 \pm 0.9$ \\
\hline
\end{tabular}

Table 2. Mean SEBT ( \pm S.D.) normalized by subject leg length.

\begin{tabular}{ccccc}
\hline & NFUB & FUB & NFB & FB \\
\hline Anterior & $0.89 \pm 0.1$ & $0.90 \pm 0.1$ & $0.88 \pm 0.1$ & $0.90 \pm 0.1$ \\
Anterior-Lateral & $0.94 \pm 0.1$ & $0.96 \pm 0.1$ & $0.93 \pm 0.1$ & $0.94 \pm 0.1$ \\
Lateral & $0.96 \pm 0.1$ & $0.97 \pm 0.1$ & $0.94 \pm 0.1$ & $0.95 \pm 0.1$ \\
Posterior-Lateral & $1.02 \pm 0.1$ & $1.03 \pm 0.1$ & $0.99 \pm 0.1$ & $1.01 \pm 0.2$ \\
Posterior & $1.01 \pm 0.1$ & $1.04 \pm 0.1$ & $1.02 \pm 0.2$ & $1.03 \pm 0.2$ \\
Posterior-Medial & $0.89 \pm 0.1$ & $0.92 \pm 0.1$ & $0.91 \pm 0.2$ & $0.95 \pm 0.2$ \\
Medial & $0.74 \pm 0.1$ & $0.76 \pm 0.1$ & $0.77 \pm 0.1$ & $0.79 \pm 0.1$ \\
Anterior-Medial & $0.80 \pm 0.1$ & $0.82 \pm 0.1$ & $0.79 \pm 0.1$ & $0.82 \pm 0.1$ \\
\hline
\end{tabular}


sults indicated the NFB was significantly less than the FB condition $(p=0.013)$. The significant difference between the extreme conditions ( $p=0.046$ ) was in favor of NFUB condition.

Range of Motion. As anticipated, the brace effectively restricted inversion and eversion upon application but had minimal effect on dorsiflexion and plantarflexion. The brace remained intact throughout the trials without adjustment by the participant or the tester. Upon re-measure of the ROM after participation it was noted that the restriction applied in all directions decreased ( $p \leq 0.05$, refer to Table 3). No other significant results were observed.

\section{DISCUSSION}

Ankle bracing is becoming increasingly more prevalent in the prevention of injuries in athletics today. During athletic events power, agility, and balance are important for optimal performance. This study investigated the effects of ankle bracing on performance during tasks focusing on the above demands before and after fatigue. Following the aforementioned exhausting conditions no significant differences between braced and non-braced conditions during the shuttle run or the vertical jump were found.

Results from these two tests partially support the hypotheses. It was hypothesized that NFB conditions would have no effect on performance. During the vertical jump, shuttle run, and SEBT performance was not significantly affected when comparing NFB. These findings help us to conclude that bracing does not affect an athlete's performance during athletic events even late in participation.

One of our hypotheses stated that FB conditions would decrease performance when compared to NFUB conditions. However, when compared to FUB, it was believed that the ankle brace would allow an individual to perform at a higher level in times of fatigue. The results did not support this hypothesis. No significant differences were found when comparing results for the vertical jump or the shuttle run. With this finding the investigators are unable to make any conclusions stating that bracing assists with power and agility performance at times of fatigued. Perhaps the fatiguing bout did not sufficiently exhaust the subject pool to a degree that the effect of

Table 3. Range of motion measurements pre-post trials ( \pm S.D).

\begin{tabular}{ccccc}
\hline & Plantarflexion & Dorsiflexion & Inversion & Eversion \\
\hline No brace & $26.3 \pm 7.8$ & $31.1 \pm 8.4$ & $30.2 \pm 11.8$ & $30.6 \pm 9.3$ \\
Brace, pre-test & $27.6 \pm 7.5$ & $30.0 \pm 9.0$ & $14.3 \pm 7.3$ & $15.6 \pm 7.0$ \\
Brace, post-test & $28.0 \pm 5.7$ & $30.6 \pm 8.2$ & $16.8 \pm 11.4$ & $20.1 \pm 12.1$ \\
\hline
\end{tabular}

bracing would be noted.

The results of the SEBT indicated that there is no significant difference when comparing braced and unbraced conditions. The significant differences were noted when comparing fatigue trials vs. non-fatigue trials in favor of fatigue. It was hypothesized that fatigue would decrease performance, but the results were just the opposite. This could have been a result of having all subjects perform non-fatigue trials prior to fatigue trials resulting in a learning effect. The only two directions with significant increases were posterior-medial and medial directions. The other six directions did not have any significant differences besides extreme conditions. In the posteriormedial and medial directions, performance was increased in the braced conditions. Ankle braces are to provide mechanical support at the joint. In doing so, the ankle brace limits eversion but primarily limits inversion. While reaching laterally during the SEBT, the planted ankle is rotated into a position of inversion; and when reaching medially the planted ankle rotates into a position of eversion. With this understanding, one would think that there would be more of a difference between $\mathrm{BF}$ and UBF when reaching laterally due to a greater limitation of inversion by the brace. This was not the case. The researchers hypothesized that the significant difference in these two directions could have been due to the fact that subjects reported these directions most challenging. The authors believe that since these were the most difficult directions, the ankle relied on the brace more for stability while fatigued than when reaching in any other direction.

Finally, it was hypothesized that FB conditions would demonstrate better control than when FUB. These data support this in the three medial directions (medial, anterior-medial, and posterior-medial) but not with the other five directions. This further supports that the ankle brace provides benefit in the more challenging directions. Although FUB results were better than FB in five of the eight directions, the differences were not significant.

The ankle brace provided adequate restriction in the directions of inversion and eversion with minimal effect to plantarflexion and dorsiflexion. The limitations to these ranges did not significantly impact performance during the shuttle run, the vertical jump or the SEBT. This supports the hypothesis that the ankle brace would effectively limit range of motion as anticipated yet would not hinder performance of agility, power and balance.

During administration of the test, subjective information was gathered from subjects to get an idea of comfort level and level of support felt by each individual. With performance of the vertical jump and shuttle run most subjects did not have any complaints, however, a few individuals stated that the brace felt limiting with the SEBT. Out of seven subjects that provided feedback on 
the SEBT during testing, five people stated that the test felt harder or they felt more off-balance during performance. Three of these individuals had worn braces in the past, two had not. Two people actually stated that they felt more support with the brace during the SEBT and both of these subjects had worn braces in the past.

As with any research project this study is not without its limitations. One limitation was not identifying previous use of ankle braces and the circumstances. Patients who had previous experience with bracing of the tested ankle may have grown accustomed to the support. This could have caused them to perform better with the brace in comparison to trials without the brace, especially in instances of chronic instability present. Chronic instability was not assessed which could have also affected our results thereby creating a second limitation. Also, our study was limited by the small sample size and testing of only healthy, active individuals. If individuals who had ankle instability were assessed and tested then results might have shown that performance with the brace is actually better than without the brace. Lastly, because of time constraints we could only test subjects on two days and therefore had to test non-fatigued before fatigued conditions. Testing fatigued conditions first would not have allowed us to test non-fatigued conditions on the same day. This limited the randomization of the testing. These limitations provide suggestions for future research with a larger sample size, patients who either have or have not worn braces in the past, and/or more randomization of the fatigue sessions.

Within our study, learning affect could have influenced results. After patients had performed one day of testing they knew what to expect when they came in for the second day, even though the bracing conditions changed. Perhaps in future studies the researchers could allot time for a practice day that will allow participants to become proficient in the tests thereby excluding the learning effect noted in the present study. This would make the testing days more about performance in the set conditions. In addition to the learning effect, we also noticed that an individual's competitive drive affected their effort. For example, after performing the first vertical jump trial many individuals with competitive drive tried to jump higher with each successive trial. Also, subjects could hear their shuttle run results and see the marks on the SEBT, which provided them with feedback regarding performance. To limit this in future studies we believe the individual needs to be blinded to their results.

\section{CONCLUSION}

Due to increased ankle injuries over the years, the use of ankle braces is becoming more prevalent. However, as the brace provides more stability at the joint, the mobility at the joint decreases potentially affecting performance.
We attempted to determine if bracing the ankle hinders an athlete's power, agility or balance before and after fatiguing the lower extremity. Under controlled testing conditions, results show that bracing does not affect an athlete's performance during athletic events even late in participation. Given more time, the researchers would have like to randomize fatigue and non-fatigue trials and incorporate an additional practice trial day to decrease learning effect. Future research should examine a variety of semi-rigid braces to determine if they have an effect on an athlete's performance. Additionally, studies could exam the difference between localized and generalized fatiguing protocols and the effect they have in similar testing conditions as this may produce different results.

\section{REFERENCES}

[1] Fong, D., Hong, Y., Chan L.K., Yung, P. and Chan, K.M. (2007) A systematic review on ankle injury and ankle sprain in sports. Sports Medicine, 37, 73-94. http://dx.doi.org/10.2165/00007256-200737010-00006

[2] Rosenbaum, D., Kamps, N., Bosch, K., Thorwestern, L., Klaus, V. and Eils, E. (2005) The influence of external ankle braces on subjective and objective parameters of performance in a sports-related agility course. Knee Surgery, Sports Traumatology, Arthroscopy, 13, 419-425. http://dx.doi.org/10.1007/s00167-004-0584-7

[3] Gross, M.T. and Liu, H.Y. (2003) The role of ankle bracing for prevention of ankle sprain injuries. Journal of Orthopedic Sports Physical Therapy, 33, 572-577. http://dx.doi.org/10.2519/jospt.2003.33.10.572

[4] Johnston, R.B., Howard, M.E., Cawley, P.W. and Losse, G.M. (1998) Effect of lower extremity muscular fatigue on motor control performance. Medicine and Science in Sports and Exercise, 30, 1703-1707. http://dx.doi.org/10.1097/00005768-199812000-00008

[5] Yaggie, J. and Armstrong, W.J. (2004) Effects of lower extremity fatigue on indices of balance. Journal of Sports Rehabilitation, 13, 312-322.

[6] Gribble, P.A., Hertel, J., Denegar, C.R. and Buckley, W.E. (2004) The effects of fatigue and chronic ankle instability on dynamic postural control. Journal of Athletic Training, 39, 321-329.

[7] Cordova, M.L., Ingersoll, C.D. and Palmieri, R.M. (2002) Efficacy of prophylactic ankle support: An experimental perspective. Journal of Athletic Training, 37, 446-457.

[8] Garrick, J.G. (1977) The frequency of injury, mechanism of injury, and epidemiology of ankle sprains. American Journal of Sports Medicine, 5, 241-242. http://dx.doi.org/10.1177/036354657700500606

[9] Garrick, J.G. and Requa, R.K. (1988) The epidemiology of foot and ankle injuries in sports. Clinical Sports Medicine, 7, 29-36.

[10] Macpherson, K., et al. (1995) Effects of a semirigid and softshell prophylactic ankle stabilizer on selected performance tests among high school football players. Journal of Orthopaedic and Sport Physical Therapy, 21, 147- 
152. http://dx.doi.org/10.2519/jospt.1995.21.3.147

[11] T2 Active Ankle Brace and Support (2009) Catalog of braces for sports and injuries.

http://supports4less.com/activeankle/anklebraces/activean kle.htm

[12] Shaw, T.R., Gribble, P.A. and Frye, J.L. (2008) Ankle bracing, fatigue, and time to stabilization in collegiate volleyball athletes. Journal of Athletic Training, 43, 164171. http://dx.doi.org/10.4085/1062-6050-43.2.164

[13] Lindley, T.R. and Kernozek, T.W. (1995) Taping and semirigid bracing may not affect ankle functional range of motion. Journal of Athletic Training, 30, 109-112.

[14] Bot, S. and van Mechelen, W. (1999) The effect of ankle bracing on athletic performance. Sports Medicine, 27, 171-
178.

http://dx.doi.org/10.2165/00007256-199927030-00003

[15] Norkin, C.C. and White, D.J. (2003) Measurment of joint motion: A guide to goniometry. 3rd Edition, F.A. Davis, Philadelphia.

[16] Brown, L.E. and Ferrigno, V.A., Eds. (2005) Training for speed, agility, and quickness. 2nd Edition, Human Kinetics, Champaign.

[17] Hardy, L., Huxel, K., Brucker, J. and Nesser, T. (2008) Prophylactic ankle braces and star excursion balance measures in healthy volunteers. Journal of Athletic Training, 43, 347-351.

http://dx.doi.org/10.4085/1062-6050-43.4.347 